\title{
Pulmonary hepatoid adenocarcinoma
}

\author{
Adil Ayub, MD, ${ }^{\mathrm{a}}$ Omar Nunez Lopez, MD, ${ }^{\mathrm{a}}$ Adam Booth, MD, ${ }^{\mathrm{b}}$ and Ikenna Okereke, $\mathrm{MD},{ }^{\mathrm{c}}$ Galveston, Tex
}

\footnotetext{
From the ${ }^{\mathrm{a}}$ Departments of Surgery, ${ }^{\mathrm{b}}$ Pathology, and ${ }^{\mathrm{c}}$ Division of Cardiothoracic Surgery, University of Texas Medical Branch, Galveston, Tex

Disclosures: Authors have nothing to disclose with regard to commercial support.

Received for publication April 11, 2019; revisions received May 23, 2019; accepted for publication June 8, 2019; available ahead of print July 26, 2019.

Address for reprints: Ikenna Okereke, MD, Division of Cardiothoracic Surgery, University of Texas Medical Branch, 301 University Blvd, Galveston, TX 77555 (E-mail: ikokerek@utmb.edu).

J Thorac Cardiovasc Surg 2019;158:e139-40

$0022-5223 / \$ 36.00$

Copyright (C) 2019 by The American Association for Thoracic Surgery

https://doi.org/10.1016/j.jtcvs.2019.06.023
}

Hepatoid adenocarcinoma (HAC) is a rare extrahepatic tumor that morphologically resembles hepatocellular carcinoma. It usually originates in the gastrointestinal tract, but can arise elsewhere. We describe our experience of a case of pulmonary HAC associated with an aggressive course and present available data on HACs utilizing the available literature and the National Cancer Institute Surveillance, Epidemiology, and End Results (SEER) database.

\section{CASE PRESENTATION}

Our patient was a 61-year-old man with a 40-pack-year smoking history and severe chronic obstructive pulmonary disease who was referred for evaluation of a suspicious lung nodule. Computed tomography scan revealed a spiculated mass measuring $2.3 \mathrm{~cm}$ in the right upper lobe. Percutaneous biopsy revealed pulmonary HAC with tumor cell positivity for thyroid transcription factor 1. An integrated whole-body positron emission tomographycomputed tomography scan showed the mass to have a standard uptake value of 4.8. No mediastinal activity was noted. Brain magnetic resonance imaging demonstrated no metastases.

He underwent video-assisted thoracoscopic right upper lobectomy with mediastinal lymph node sampling. Final pathologic review revealed a moderately differentiated, pulmonary HAC (Figure 1). The tumor stained positive for thyroid transcription factor 1, hepatocyte paraffin antigen-1, cytokeratin 7 , cytokeratin 8 , carcinoembryonic antigen, and cytokeratin 19, and was negative for alpha fetoprotein (AFP), p40, napsin, and cluster of differentiation 10. Final pathological stage was pT1b N0 M0.

Within 3 months after surgery he developed increasing pain in his pelvis, back, and neck. MRI of the spine and pelvis showed diffuse bony disease in multiple areas in the spine and sacrum. A biopsy of the sacral lesion showed metastatic HAC. The patient received radiation therapy to the symptomatic bony metastases but developed progressive weakness and was ultimately transferred to hospice care. He died 6 months after diagnosis.

\section{DISCUSSION}

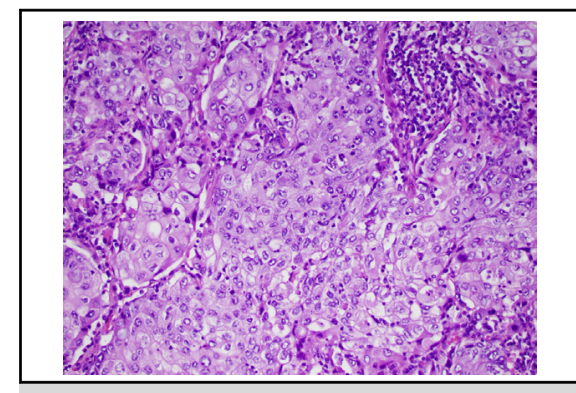

High-powered image of pulmonary hepatoid adenocarcinoma.

\section{Central Message}

Pulmonary hepatoid adenocarcinoma is a rare and aggressive tumor with poor overall prognosis. Before surgery, a comprehensive workup is required to ensure no metastatic disease is present.

See Commentary on page e141.

Extrahepatic HACs are described as rare and aggressive tumors most commonly originating in the gastric region $(63 \%){ }^{1}$ Histologically, these tumors appear as lightly acidophilic cells with abundant cytoplasm and can be diagnosed with fine needle aspiration. HACs follow an aggressive clinical course with a reported overall median survival of 11 months (range, 1-116 months) and a 1-year survival rate of $55 \%{ }^{2}$ Primary pulmonary HACs are

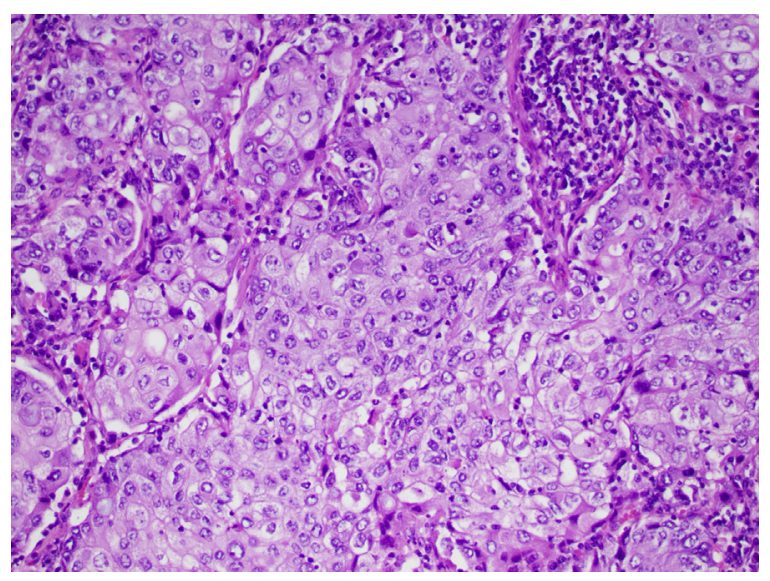

FIGURE 1. Pulmonary hepatoid adenocarcinoma with a predominantly acinar pattern morphologically resembling hepatocellular carcinoma. Tumor cells form glands and nests with pale eosinophilic cytoplasm and moderate nuclear pleomorphism (hematoxylin and eosin stain $\times 20$ ). 
TABLE 1. Patient characteristics based on the National Cancer Institute Surveillance, Epidemiology, and End Results database from 1988 to $2014(\mathrm{~N}=41)$

\begin{tabular}{lc}
\hline \multicolumn{1}{c}{ Characteristic } & Result \\
\hline Age at diagnosis (y) & $63.6 \pm 12.0$ \\
Male & $24(58.5)$ \\
White & $34(82.9)$ \\
Tumor size (cm) & $8.2 \pm 12.5$ \\
Stage at diagnosis & \\
I & $3(7.3)$ \\
II & $4(9.8)$ \\
III & $5(12.2)$ \\
IV & $26(63.4)$ \\
Unknown & $3(7.3)$ \\
Tumor location & \\
Right upper lobe & $12(29.3)$ \\
Left upper lobe & $11(26.8)$ \\
Left lower lobe & $5(12.2)$ \\
Right lower lobe & $2(4.9)$ \\
Not specified & $11(26.8)$ \\
Surgical resection & \\
Yes & $5(12.2)$ \\
No & $36(87.8)$ \\
\hline
\end{tabular}

Values are presented as mean \pm standard deviation or $\mathrm{n}(\%)$.

extremely rare, with approximately 36 cases reported in the literature. According to previous reports, pulmonary HACs show a predominance in men $(92 \%)$ and usually present as stage III or stage IV disease. ${ }^{3}$ They are mostly seen in heavy smokers. In general, these tumors are quite large at the time of diagnosis, are usually diagnosed at an advanced stage, and are associated with poor outcomes. ${ }^{4}$

We queried the SEER database from 1988 to 2014. Using this database, we identified 41 cases of primary pulmonary HAC (Table 1). Mean age was 63.6 years. Mean tumor size was $8.2 \mathrm{~cm}$. Eighty-two percent of tumors were stage III or IV at presentation. Median overall survival for the entire cohort was 5 months $(95 \%$ confidence interval, 3.1-6.9 months), with 1- and 3-year survival rates of only $35 \%$ and $14 \%$, respectively.

Our report and review of the literature is the largest to date concerning pulmonary HACs. Although previous literature has also suggested that this tumor usually has an aggressive clinical course, prognostic indicators have been difficult to identify. The significance of an elevated AFP level is unknown. The majority of cases have demonstrated elevated serum AFP and/or positive AFP expression on histopathologic analysis. Lack of expression of AFP does not appear to be reliably associated with better outcomes. In our patient, serum AFP levels were not obtained, and the tumor showed no expression of AFP.

There is no standard treatment for HAC. Although advanced disease will usually be treated by platinumbased chemotherapy and/or radiation treatment, patients with localized disease should undergo surgical resection. ${ }^{5}$ It appears that the poor overall survival is a result of the advanced stage at diagnosis for most patients. Mediastinal tissue sampling should be considered before surgery because so many patients present with advanced disease, but there is no available evidence about how frequently this sampling will lead to upstaging. Clinicians should be aware that HACs will tend to have a very aggressive course and a much worse behavior than most non-small cell lung cancers.

\section{CONCLUSIONS}

Primary lung HACs are rare tumors with limited data concerning clinical presentation, management, and outcomes. Available literature and a SEER database analysis show that this tumor generally has an extremely poor prognosis. A comprehensive workup and accurate diagnosis is important before any consideration of surgery.

\section{References}

1. Metzgeroth G, Strobel P, Baumbusch T, Reiter A, Hastka J. Hepatoid adenocarcinoma-review of the literature illustrated by a rare case originating in the peritoneal cavity. Onkologie. 2010;33:263-9.

2. Haninger DM, Kloecker GH, Bousamra IM, Nowacki MR, Sloane SP. Hepatoid adenocarcinoma of the lung: report of five cases and review of the literature. Mod Pathol. 2014;27:535-42.

3. Grossman K, Beasley MB, Braman SS. Hepatoid adenocarcinoma of the lung: review of a rare form of lung cancer. Respir Med. 2016;119:175-9.

4. Papatsimpas G, Kamposioras K, Goula K, Papaparaskeva K, Loukides S, Kotoulas C, et al. Hepatoid pancoast tumor. A case report and review of the literature. Lung Cancer. 2012;77:239-45.

5. Che YQ, Wang S, Luo Y, Wang J, Wang L. Hepatoid adenocarcinoma of the lung: presenting mediastinal metastasis without transfer to the liver. Oncol Lett. 2014;8: 105-10. 